\title{
Epidemiology of carbapenem-resistant Enterobacteriaceae: a 5-year experience at a tertiary care hospital
}

This article was published in the following Dove Medical Press journal: Infection and Drug Resistance

\author{
Darunee Chotiprasitsakul' \\ Sirawat Srichatrapimuk ${ }^{2}$ \\ Suppachok Kirdlarp' \\ Alexander D Pyden ${ }^{3}$ \\ Pitak Santanirand ${ }^{4}$ \\ 'Division of Infectious Diseases, \\ Department of Medicine, Faculty \\ of Medicine Ramathibodi Hospital, \\ Mahidol University, Bangkok, \\ Thailand; ${ }^{2}$ Chakri Naruebodindra \\ Medical Institute, Faculty of Medicine \\ Ramathibodi Hospital, Mahidol \\ University, Samutprakan, Thailand; \\ ${ }^{3}$ Department of Pathology, Beth Israel \\ Deaconess Medical Center, Harvard \\ Medical School, Boston, MA, USA; \\ ${ }^{4}$ Clinical Microbiology Laboratory, \\ Department of Pathology, Faculty \\ of Medicine Ramathibodi Hospital, \\ Mahidol University, Bangkok, Thailand
}

Correspondence: Darunee Chotiprasitsakul

Division of Infectious Diseases, Department of Medicine, Faculty of Medicine Ramathibodi Hospital, Mahidol University, 270 Rama VI Road, Ratchathewi, Bangkok 10400, Thailand

Tel +66220 I I58I

Fax +6622012232

Email darunee.cho@mahidol.ac.th
Purpose: The incidence of carbapenem-resistant Enterobacteriaceae (CRE) has been increasing worldwide. Ertapenem resistance is mediated by non-carbapenemase mechanisms, and has less of an effect on susceptibility to imipenem and meropenem. This study aimed to study the epidemiology of CRE, and to compare risk factors and related mortality between nonsusceptibility to ertapenem alone Enterobacteriaceae (NSEE), with non-susceptibility to other carbapenems (imipenem, meropenem, or doripenem) Enterobacteriaceae (NSOCE) at a tertiary care hospital in Thailand.

Methods: All CRE isolated were identified between December 2011 and December 2016. Quarterly incidence rate was estimated. Hospital-wide carbapenem consumption was calculated as defined daily doses (DDD). Relationships between hospital-wide carbapenem consumption and incidence of CRE were tested. Factors associated with NSEE and NSOCE, and risk factors associated with 14- and 30-day mortality in patients with CRE infection were determined. Results: The quarterly CRE incidence increased significantly from 3.37 per 100,000 patientdays in the last quarter of 2011 to 32.49 per 100,000 patient-days in the last quarter of 2016. ( $P$ for trend $<0.001$ ). Quarterly hospital-wide carbapenem consumption increased 1.58 DDD per 1,000 patient-days ( $P$ for trend $=0.004$ ). The Poisson regression showed the expected increase of CRE incidence was 1.02 per 100,000 patient-days for a 1 DDD per 1,000 patient-days increase in carbapenem consumption $(P<0.001)$. There were 40 patients with NSEE and 134 patients with NSOCE in the 5-year study period. The NSEE group had significantly lower carbapenem exposure compared with the NSOCE group (adjusted odds ratio: $0.25 ; P=0.001$ ). No difference in 14-day and 30-day all-cause mortality between the two groups was observed.

Conclusion: The incidence of CRE has risen significantly at our institution. Previous carbapenem use was associated with NSOCE. This hospital-wide carbapenem use was significantly associated with the increasing incidence of CRE.

Keywords: CRE, risk factors, incidence, carbapenems, antimicrobial stewardship

\section{Introduction}

Carbapenem-resistant Enterobacteriaceae (CRE) are an urgent global public health problem. CRE infections are associated with high mortality and have limited available effective treatment. ${ }^{1-3}$ The mortality of CRE bloodstream infection was $39 \%$ in patients receiving appropriate treatment and $61 \%$ in patients receiving inappropriate treatment in a retrospective international cohort study conducted in ten countries. ${ }^{4}$ The growing prevalence of CRE has been reported worldwide. ${ }^{1,5-7}$ A surveillance of CRE among 12,741 isolates of Enterobacteriaceae at a 2,300-bed university hospital in Bangkok, Thailand reported CRE prevalence of $1.4 \%$ during a period from 
2009 to $2011 .^{8}$ Of the 4,818 Enterobacteriaceae screening isolates, the CRE prevalence was $2.2 \%$, and eight isolates carried carbapenemase genes in a study conducted at a university hospital in Khon Kaen, Thailand between 2010 and 2011.9 At our institution, CRE was first detected in December 2011. We have monitored and tracked all cases of carbapenem-non-susceptible Enterobacteriaceae since then. Despite standard infection control measures and particularly strict contact precautions, cases of CRE have substantially increased at our institution. Carbapenemases are beta-lactamase enzymes that inactivate carbapenems, and other beta-lactam antibiotics. This enzyme-mediated resistance is clinically important, as it confers high levels of carbapenem minimum inhibitory concentrations (MICs). ${ }^{10}$ However, ertapenem resistance in Enterobacteriaceae was mainly associated with beta-lactamases, consisting of AmpC cephalosporinases and/or extended-spectrum beta-lactamase, combined with porin deficiency. ${ }^{11,12}$ Most of these strains were resistant to ertapenem, while still moderately susceptible to imipenem and meropenem. ${ }^{11}$ We aim to describe CRE epidemiology at our institution, in a resource-limited setting, and to compare the phenotype of carbapenem non-susceptibility to ertapenem alone Enterobacteriaceae (NSEE) with non-susceptibility to other carbapenems (imipenem, meropenem, or doripenem) Enterobacteriaceae (NSOCE) in terms of risk factors and mortality.

\section{Materials and methods Study population}

This retrospective cohort study was conducted at Ramathibodi Hospital, a 1,166-bed tertiary-care medical center with kidney and stem cell transplant units in Bangkok, Thailand between December 2011 and December 2016. All CRE isolated from clinical and surveillance cultures were identified from daily microbiology laboratory studies and reported to infection control units. Patients 18 years of age and older with CRE were enrolled into the comparative study.

This study was approved by the ethics institutional review boards of Ramathibodi hospital, with a waiver of informed consent.

\section{Data collection}

Demographic information, pre-existing medical conditions, intensive care unit (ICU) admission, Sequential Organ Failure Assessment (SOFA) score, mechanical ventilation, surgery, CRE colonization or infection status, microbiologi- cal, treatment, and mortality data for eligible patients were retrieved from medical records. An incident CRE case was defined as the first CRE isolate from a patient during the study period. Each patient was reviewed from 90 days prior to 90 days after the first CRE detection. Independent adjudication of patients with CRE growing in clinical cultures to determine colonization or infection status and mortality attributable to CRE was performed by two infectious diseases physicians and one physician in infectious diseases training. CRE infection was defined as CRE in blood culture; CRE isolated in sputum cultures with symptoms and signs of pneumonia, and the presence of an infiltrate on chest imaging; CRE isolated from intra-abdominal fluid, skin, soft tissue, bone, or joints with symptoms and signs of infection. A urine culture was considered to represent infection if positive with $\geq 10^{5}$ colony-forming units (CFU)/ $\mathrm{mL}$ with no more than two species of organisms in patients with symptoms and signs of urinary tract infection, or with no other explainable cause of fever.

All-cause mortality was assessed at 14 and 30 days after the diagnosis of CRE infections in patients who received antibiotic treatment for at least 3 days.

\section{Antimicrobial susceptibility testing}

All Enterobacteriaceae isolates were tested for their susceptibility to carbapenems and other antimicrobial agents using automated microbroth dilution testing systems (Sensititre ${ }^{\mathrm{TM}}$; Thermo Fisher Scientific, Cleveland, OH, USA). The MIC values were interpreted according to the Clinical and Laboratory Standards Institute (CLSI) cutoff levels. ${ }^{13}$ Carbapenem non-susceptibility was identified when MIC was $>1 \mathrm{mg} / \mathrm{L}$ for imipenem, meropenem, doripenem, and $>0.5 \mathrm{mg} / \mathrm{L}$ for ertapenem.

\section{Statistical analysis}

To calculate the quarterly incidence rate from clinical and surveillance cultures of CRE, patient-days was used as the denominator. Hospital-wide carbapenem consumption were collected and calculated as defined daily doses (DDD) per 1,000 patient-days. The trend over time was estimated using a linear regression model, with time as the independent variable. Relationships between hospital-wide carbapenem consumption and incidence of CRE were tested using Poisson regression.

Comparative analysis of factors associated with NSEE and NSOCE was conducted. Chi square or Fisher's exact tests were used to compare categorical variables, as appropriate. Wilcoxon rank-sum tests were used to compare continuous 
variables. Logistic regression was used to estimate the odds ratios (ORs) and associated 95\% CIs for factors associated with NSEE. Cox proportional hazards modeling was used to estimate the unadjusted hazard ratios (HRs) and associated 95\% confidence intervals (CIs) for potential risk factors associated with 14- and 30-day mortality in patients with CRE infection who received antibiotic treatment at least 3 days following the onset of infection. Variables yielding $P$-values $<0.10$ on univariate analysis, or with clinical plausibility, were included in multivariate models to identify independent predictors of mortality. The proportional hazards assumption was examined by graphically inspecting complementary $\log -\log$ plots for each variable. Collinearity was checked with various inflation factors. A two-sided $P$-value of less than 0.05 was considered statistically significant for all tests. Statistical analysis was performed using Stata, version 12.0 (Stata Corp., College Station, TX, USA).

\section{Results}

\section{Incidence of CRE}

The quarterly CRE incidence of unique patients increased significantly from 3.37 per 100,000 patient-days in the last quarter of 2011 to 32.49 per 100,000 patient-days in the last quarter of 2016. Quarterly CRE incidence increased 1.07 per 100,000 patient-days $(95 \% \mathrm{CI}=0.69-1.46$; $P$-value for trend $<0.001)$. Quarterly hospital-wide carbapenem consumption increased 1.58 DDD per 1,000 patient-days (95\%
$\mathrm{CI}=0.56-2.59 ; P$-value for trend=0.004). The Poisson regression showed the expected increase of CRE incidence was 1.02 per 100,000 patient-days for a 1 DDD per 1,000 patient-days increase in carbapenem consumption $(95 \% \mathrm{CI}=1.01-1.03$; $P$-value $<0.001$ ) (Figure 1).

\section{Clinical characteristics of patients with NSEE and NSOCE}

A cohort of 199 unique patients with CRE isolates were identified during the study period. After exclusion of 25 pediatric patients, 174 adult patients were included in the comparative analysis. There were 40 patients with NSEE and 134 patients with NSOCE. A comparison of baseline characteristics between NSEE and NSOCE patients is shown in Table 1. The median duration from admission to first CRE detection in NSEE group and NSOCE group was 22 days (interquartile range $[\mathrm{IQR}]=12-30$ days) and 19 days ( $\mathrm{IQR}=10-49$ days), respectively. Several differences between the two groups were evident. Patients with NSEE were more likely to have intra-abdominal infection. Patients with NSOCE had higher SOFA scores, higher rates of admission to the ICU, more likelihood to be on mechanical ventilation at the time of first CRE isolation, and more exposure to carbapenems during the preceding 90 days. In the multivariate analysis, lower carbapenem exposure was significantly associated with the NSEE group $(\mathrm{OR}=0.25$; 95\% CI=0.11-0.56) (Table 1).

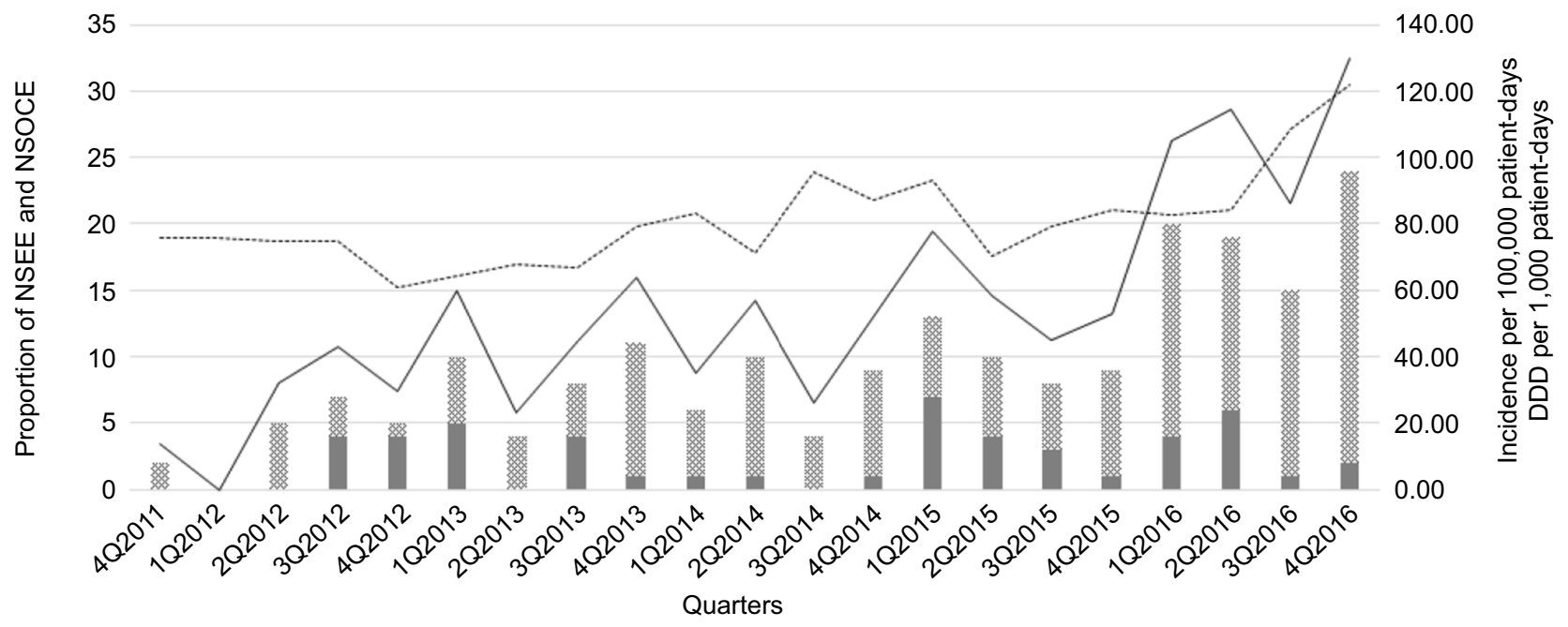

NSEE Incidence of CRE

........ Hospital-wide carbapenem consumption

Figure I Quarterly incidence rate of all CRE (solid line) and hospital-wide carbapenem consumption (dashed line) from quarter 0 (October 20 I I) to quarter 2 I (ending December 2016). Bar charts represent relative proportions of NSEE and NSOCE.

Abbreviations: CRE, carbapenem-resistant Enterobacteriaceae; DDD, defined daily doses; NSEE, carbapenem non-susceptibility to ertapenem alone Enterobacteriaceae; NSOCE, non-susceptibility to other carbapenems (imipenem, meropenem, or doripenem) Enterobacteriaceae. 
Table I Baseline characteristics of 174 adult patients with CRE and comparative analysis of NSEE and NSOCE

\begin{tabular}{|c|c|c|c|c|c|c|c|}
\hline \multirow[b]{2}{*}{ Variables } & \multirow[b]{2}{*}{$\begin{array}{l}\text { NSEE } \\
(\mathrm{N}=40)\end{array}$} & \multirow[b]{2}{*}{$\begin{array}{l}\text { NSOCE } \\
(N=134)\end{array}$} & \multirow[b]{2}{*}{$P$-value } & \multicolumn{2}{|c|}{ Univariate analysis } & \multicolumn{2}{|c|}{ Multivariate analysis ${ }^{a}$} \\
\hline & & & & OR $(95 \% \mathrm{Cl})$ & $P$-value & OR $(95 \% \mathrm{Cl})$ & P-value \\
\hline Age (years), median (IQR) & $64(55-73)$ & $71(54-78)$ & 0.24 & $0.99(0.97-1.01)$ & 0.40 & & \\
\hline Male & $21(52.5 \%)$ & $58(43.3 \%)$ & 0.30 & $0.69(0.34-1.40)$ & 0.31 & & \\
\hline Previous admission within 90 days & & & 0.59 & & & & \\
\hline No previous admission & $20(50.0 \%)$ & $63(47.0 \%)$ & & & & & \\
\hline Same hospital & $15(37.5 \%)$ & $60(44.8 \%)$ & & & & & \\
\hline Outside hospital & $5(12.5 \%)$ & II (8.2\%) & & & & & \\
\hline \multicolumn{8}{|l|}{ Pre-existing medical conditions } \\
\hline Chronic pulmonary disease & $0(0 \%)$ & $9(6.7 \%)$ & 0.12 & $0.16(0.01-2.86)$ & 0.21 & & \\
\hline Cardiovascular disease & $8(20.0 \%)$ & $30(22.4 \%)$ & 0.75 & $0.87(0.36-2.08)$ & 0.75 & & \\
\hline Chronic liver disease & $0(0 \%)$ & $7(5.2 \%)$ & 0.35 & $0.21(0.01-3.76)$ & 0.29 & & \\
\hline Chronic kidney disease & $5(12.5 \%)$ & $18(13.4 \%)$ & 0.88 & $0.92(0.32-2.66)$ & 0.88 & & \\
\hline Neurologic disease & $3(7.5 \%)$ & $21(15.7 \%)$ & 0.29 & $0.44(0.12-1.55)$ & 0.20 & & \\
\hline Diabetes mellitus & II (27.5\%) & $49(36.6 \%)$ & 0.29 & $0.66(0.30-1.43)$ & 0.29 & & \\
\hline Solid tumor & $18(45.0 \%)$ & $45(33.6 \%)$ & 0.19 & $1.62(0.79-3.32)$ & 0.19 & & \\
\hline Hematologic malignancies & $4(10.0 \%)$ & $15(11.2 \%)$ & $>0.99$ & $0.88(0.28-2.82)$ & 0.83 & & \\
\hline HIV & $0(0 \%)$ & $2(1.5 \%)$ & $>0.99$ & & & & \\
\hline Chemotherapy in 6 months & $6(15.0 \%)$ & $17(12.7 \%)$ & 0.71 & $1.21(0.44-3.32)$ & 0.71 & & \\
\hline $\begin{array}{l}\text { Immunomodulatory therapy or } \\
\text { corticosteroids for } \geq 14 \text { days }\end{array}$ & $3(7.5 \%)$ & $12(9.0 \%)$ & $>0.99$ & $0.82(0.22-3.08)$ & 0.77 & & \\
\hline SOFA score, median (IQR) & $2(I-5)$ & $4(2-7)$ & 0.007 & $0.88(0.79-0.98)$ & 0.02 & $0.98(0.87-1.1 \mathrm{I})$ & 0.77 \\
\hline \multicolumn{8}{|l|}{ Epidemiological risks } \\
\hline $\begin{array}{l}\text { Time from admission to CRE detection, } \\
\text { days, median (IQR) }\end{array}$ & $22(12-30)$ & $19(10-49)$ & 0.64 & $0.99(0.98-1.00)$ & 0.13 & & \\
\hline In ICU at the time of culture & $7(17.5 \%)$ & $53(39.6 \%)$ & 0.01 & $0.32(0.13-0.79)$ & 0.01 & $0.39(0.13-1.16)$ & 0.09 \\
\hline On mechanical ventilation & $13(32.5 \%)$ & $80(59.7 \%)$ & 0.002 & $0.33(0.15-0.69)$ & 0.003 & $0.63(0.24-1.64)$ & 0.35 \\
\hline Central venous line & $7(17.5 \%)$ & $26(19.4 \%)$ & 0.79 & $0.88(0.35-2.21)$ & 0.79 & & \\
\hline Hemodialysis & $6(15.0 \%)$ & $29(21.6 \%)$ & 0.36 & $0.64(0.24-1.67)$ & 0.36 & & \\
\hline Surgery in the preceding 90 days & $26(65.0 \%)$ & $70(52.2 \%)$ & 0.15 & $1.70(0.82-3.53)$ & 0.16 & & \\
\hline \multicolumn{8}{|c|}{ Co-colonization with other MDROs during preceding 90 days } \\
\hline ESBL Gram-negative & II (27.5\%) & $56(4 I .8 \%)$ & 0.10 & $0.53(0.24-1.15)$ & 0.11 & & \\
\hline Acinetobacter baumannii & $3(7.5 \%)$ & $21(15.7 \%)$ & 0.29 & $0.44(0.12-1.55)$ & 0.2 & & \\
\hline Pseudomona aeruginosa & $0(0 \%)$ & $8(6.0 \%)$ & 0.21 & $0.18(0.01-3.25)$ & 0.25 & & \\
\hline MRSA & $0(0 \%)$ & $\mathrm{II}(8.2 \%)$ & 0.07 & $0.13(0.01-2.30)$ & 0.17 & & \\
\hline VRE & $\mathrm{I}(2.5 \%)$ & $3(2.2 \%)$ & $>0.99$ & $1.12(0.1 \mathrm{I}-1 \mathrm{I} .07)$ & 0.92 & & \\
\hline \multicolumn{8}{|c|}{ Previous antibiotic exposure within 90 days } \\
\hline Carbapenems & $15(37.5 \%)$ & $93(69.4 \%)$ & $<0.001$ & $0.26(0.13-0.55)$ & $<0.001$ & $0.25(0.11-0.56)$ & 0.001 \\
\hline Ceftriaxone & $12(30.0 \%)$ & $5 I(38.1 \%)$ & 0.35 & $0.70(0.33-1.49)$ & 0.35 & & \\
\hline Cefepime & $4(10.0 \%)$ & $12(9.0 \%)$ & 0.76 & $1.13(0.34-3.72)$ & 0.84 & & \\
\hline Piperacillin/tazobactam & $15(37.5 \%)$ & $5 I(38.1 \%)$ & 0.95 & $0.98(0.47-2.02)$ & 0.95 & & \\
\hline Fluoroquinolones & $14(35.0 \%)$ & $36(26.9 \%)$ & 0.32 & $\mathrm{I} .47(0.69-3 . \mathrm{II})$ & 0.32 & & \\
\hline Aminoglycosides & $2(5.0 \%)$ & $12(9.0 \%)$ & 0.53 & $0.54(0.11-2.50)$ & 0.43 & & \\
\hline \multicolumn{8}{|l|}{ Type of identification } \\
\hline Colonization & $18(45.0 \%)$ & $62(46.3 \%)$ & 0.89 & $0.95(0.47-1.93)$ & 0.89 & & \\
\hline Primary bacteremia & $\mathrm{I}(2.5 \%)$ & $14(10.5 \%)$ & 0.20 & $0.22(0.03-1.73)$ & 0.15 & & \\
\hline Pneumonia & $\mathrm{I}(2.5 \%)$ & $12(9.0 \%)$ & 0.30 & $0.26(0.03-2.07)$ & 0.20 & & \\
\hline Skin and soft tissue infection & $3(7.5 \%)$ & $8(6.0 \%)$ & 0.72 & $1.28(0.32-5.06)$ & 0.73 & & \\
\hline Urinary tract infection & $5(12.5 \%)$ & $19(14.2 \%)$ & 0.79 & $0.86(0.30-2.48)$ & 0.79 & & \\
\hline Intra-abdominal infection & $12(30.0 \%)$ & $21(15.7 \%)$ & 0.04 & $2.30(1.01-5.24)$ & 0.046 & $2.46(0.96-6.31)$ & 0.06 \\
\hline
\end{tabular}

Note: adjusted for variables with $P<0.10$ in univariate analysis.

Abbreviations: CRE, carbapenem-resistant Enterobacteriaceae; ESBL, extended spectrum beta-lactamases; HIV, human immunodeficiency virus; ICU, intensive care unit; IQR, interquartile range; MDROs, multidrug-resistant organisms; MRSA, methicillin-resistant Staphylococcus aureus; OR, odds ratio; SOFA, Sequential Organ Failure Assessment; VRE, vancomycin-resistant enterococci. 
Microorganisms and antibiotic susceptibility of NSEE and NSOCE

The most common CRE organisms isolated in the NSOCE group were Klebsiella pneumoniae (70.7\%) followed by Escherichia coli (14.0\%) and Enterobacter species (12.7\%). Among the NSEE group, the most common CRE organisms isolated were Enterobacter species (38.8\%) followed by $E$ coli (32.7\%) and K. pneumoniae (28.6\%). The most prevalent sources of CRE detection were urine, followed by intra-abdominal specimen in both groups.

The susceptibility rates to carbapenems in the NSOCE group were as follows: imipenem (23.3\%), meropenem (4.0\%), and doripenem (15.5\%). The susceptibility rates to amikacin in the NSEE group and NSOCE groups were $81.6 \%$ and $64.9 \%$, respectively. Overall, susceptibility rates to colistin and tigecycline were approximately $90 \%$ in both groups (Table 2).

\section{Analysis of risk factors associated with 14-day and 30-day all-cause mortality in CRE-infected patients}

Twenty-two patients (55.0\%) in the NSEE group and 69 patients $(51.5 \%)$ in the NSOCE group received antibiotic treatment for CRE infection. Of these, 16 patients $(72.7 \%)$ in the NSEE group and 48 patients (69.5\%) in the NSOCE group received in vitro active empiric treatment within the first 3 days after the onset of CRE infection. Nine patients $(40.9 \%)$ in the NSEE group and 16 patients $(23.2 \%)$ in the NSOCE group received monotherapy with imipenem or meropenem. Seven of these 16 patients $(43.8 \%)$ in the NSOCE group received active carbapenem treatment based on in vitro susceptibility testing. The 14-day all-cause mortality in the NSEE group and the NSOCE group with CRE infection were $4.6 \%$ and $11.6 \%$, respectively. No difference in 14-day all-cause mortality between the NSEE group and NSOCE group was observed in the univariate analysis (HR=0.37; 95\% CI=0.05-2.94). The 30-day all-cause mortality in the NSEE group and NSOCE group were $9.1 \%$ and $23.2 \%$, respectively ( $\mathrm{HR}=0.43 ; 95 \% \mathrm{CI}=0.10-1.90)$. Factors significantly associated with 30 -day all-cause mortality in the multivariate model were chronic kidney disease, hematologic malignancies, and SOFA score (Table 3).

\section{Discussion}

The incidence of CRE clinical cultures at our institution continuously increased from 3.37 per 100,000 patient-days in the last quarter of 2011, to 7.37 per 100,000 patient-days in the last quarter of 2012, and 15.98 per 100,000 patient-days in the
Table 2 Microorganisms and antibiotic susceptibility profiles of NSEE and NSOCE isolates, December 20II to December 2016

\begin{tabular}{|c|c|c|}
\hline & $\begin{array}{l}\text { NSEE } \\
(N=49)\end{array}$ & $\begin{array}{l}\text { NSOCE } \\
(N=150)\end{array}$ \\
\hline \multicolumn{3}{|l|}{ Bacterial species } \\
\hline Klebsiella pneumoniae & $14(28.6 \%)$ & $106(70.7 \%)$ \\
\hline Escherichia coli & $16(32.7 \%)$ & $21(14.0 \%)$ \\
\hline Enterobacter spp. & $19(38.8 \%)$ & $19(12.7 \%)$ \\
\hline $\begin{array}{l}\text { Other (Citrobacter spp., Proteus spp., } \\
\text { Serratia marcescens) }\end{array}$ & $0(0 \%)$ & $4(2.7 \%)$ \\
\hline \multicolumn{3}{|l|}{ Source } \\
\hline Urine & $19(38.8 \%)$ & $69(46.0 \%)$ \\
\hline Intra-abdominal & $16(32.7 \%)$ & $32(21.3 \%)$ \\
\hline Respiratory specimens & $6(12.2 \%)$ & 17 (II.3\%) \\
\hline Wound swab & $6(12.2 \%)$ & $18(12.0 \%)$ \\
\hline Blood & $2(4.1 \%)$ & $14(9.3 \%)$ \\
\hline Amikacin (no. of isolates tested) & 49 & 148 \\
\hline Susceptible, $n$ & $40(81.6 \%)$ & $96(64.9 \%)$ \\
\hline Intermediate, $\mathrm{n}$ & $2(4.1 \%)$ & $\mathrm{I}(0.7 \%)$ \\
\hline Resistant, $\mathrm{n}$ & $7(14.3 \%)$ & $5 \mathrm{I}(34.5 \%)$ \\
\hline Gentamicin (no. of isolates tested) & 49 & 148 \\
\hline Susceptible, $n$ & $22(44.9 \%)$ & $56(37.8 \%)$ \\
\hline Intermediate, $\mathrm{n}$ & $\mathrm{I}(2.0 \%)$ & $5(3.4 \%)$ \\
\hline Resistant, $\mathrm{n}$ & $26(53.1 \%)$ & $87(58.8 \%)$ \\
\hline Colistin (no. of isolates tested) & 49 & 148 \\
\hline Susceptible, $\mathrm{n}$ & $46(93.9 \%)$ & $137(92.6 \%)$ \\
\hline Intermediate, $\mathrm{n}$ & $0(0 \%)$ & $0(0 \%)$ \\
\hline Resistant, $\mathrm{n}$ & $3(6.1 \%)$ & II (7.4\%) \\
\hline Tigecycline (no. of isolates tested) & 49 & 148 \\
\hline Susceptible, $n$ & $44(89.8 \%)$ & $135(91.2 \%)$ \\
\hline Intermediate, $\mathrm{n}$ & $3(6.1 \%)$ & $7(4.7 \%)$ \\
\hline Resistant, $\mathrm{n}$ & $2(4.1 \%)$ & $6(4.1)$ \\
\hline Imipenem (no. of isolates tested) & 49 & 150 \\
\hline Susceptible, $n$ & $49(100 \%)$ & $35(23.3 \%)$ \\
\hline Intermediate, $\mathrm{n}$ & $0(0 \%)$ & $20(13.3 \%)$ \\
\hline Resistant, $\mathrm{n}$ & $0(0 \%)$ & $95(63.3 \%)$ \\
\hline Meropenem (no. of isolates tested) & 49 & 150 \\
\hline Susceptible, $n$ & $49(100 \%)$ & $6(4.0 \%)$ \\
\hline Intermediate, $\mathrm{n}$ & $0(0 \%)$ & $18(12.0 \%)$ \\
\hline Resistant, $\mathrm{n}$ & $0(0 \%)$ & $126(84.0 \%)$ \\
\hline Doripenem (no. of isolates tested) & 49 & 148 \\
\hline Susceptible, $n$ & $49(100 \%)$ & $23(15.5 \%)$ \\
\hline Intermediate, $\mathrm{n}$ & $0(0 \%)$ & 27 (I8.2\%) \\
\hline Resistant, $\mathrm{n}$ & $0(0 \%)$ & $98(66.2 \%)$ \\
\hline $\begin{array}{l}\text { Piperacillin/tazobactam (no. of } \\
\text { isolates tested) }\end{array}$ & 49 & 147 \\
\hline Susceptible, $n$ & $8(16.3 \%)$ & $6(4.1 \%)$ \\
\hline Intermediate, $\mathrm{n}$ & $4(8.2 \%)$ & $10(6.8 \%)$ \\
\hline Resistant, $\mathrm{n}$ & 37 (75.5\%) & $|3|(89.1 \%)$ \\
\hline $\begin{array}{l}\text { Amoxicillin/clavulanate (no. of } \\
\text { isolates tested) }\end{array}$ & 49 & 145 \\
\hline Susceptible, $n$ & $3(6.1 \%)$ & $\mathrm{I}(0.7 \%)$ \\
\hline Intermediate, $\mathrm{n}$ & $9(18.4 \%)$ & II (7.6\%) \\
\hline Resistant, $\mathrm{n}$ & 37 (75.5\%) & 133 (91.7\%) \\
\hline $\begin{array}{l}\text { Ciprofloxacin (no. of isolates } \\
\text { tested) }\end{array}$ & 49 & 148 \\
\hline Susceptible, $n$ & 9 (18.4\%) & $15(10.1 \%)$ \\
\hline Intermediate, $\mathrm{n}$ & $2(4.1 \%)$ & $6(4.1 \%)$ \\
\hline Resistant, $\mathrm{n}$ & $28(57.1 \%)$ & $127(85.8 \%)$ \\
\hline
\end{tabular}

(Continued) 
Table 2 (Continued)

\begin{tabular}{|l|l|l|}
\hline & $\begin{array}{l}\text { NSEE } \\
(\mathbf{N}=49)\end{array}$ & $\begin{array}{l}\text { NSOCE } \\
(\mathbf{N}=150)\end{array}$ \\
\hline Levofloxacin (no. of isolates tested) & 49 & 146 \\
\hline Susceptible, $n$ & $\mathrm{II}(22.4 \%)$ & $23(15.8 \%)$ \\
\hline Intermediate, $\mathrm{n}$ & $5(10.2 \%)$ & $10(6.8 \%)$ \\
\hline Resistant, $\mathrm{n}$ & $33(67.3 \%)$ & $\mathrm{I} 13(77.4 \%)$ \\
\hline
\end{tabular}

Abbreviations: NSEE, carbapenem nonsusceptibility to ertapenem alone Enterobacteriaceae; NSOCE, non-susceptibility to other carbapenems (imipenem, meropenem, or doripenem) Enterobacteriaceae.

last quarter of 2013. This was relatively high compared to the reported incidence of CRE clinical cases of 2.94 per 100,000 population during $2012-2013$ in the US, ${ }^{1}$ and $2.89-10.32$ per 100,000 patient-days during 2010-2013 in Singapore. ${ }^{5}$ Furthermore, our incidence of CRE exponentially increased almost ten-fold to 32.49 per 100,000 patient-days in 2016 . The standard infection control and prevention measures could not slow down the spread of CRE at our institution. More proactive infection prevention strategies are required to contain CRE. Almost $71 \%$ of NSOCE in our study were $K$. pneumoniae. This is consistent with a previous study in Thailand which found carbapenem-resistant $K$. pneumoniae (CRKP) isolates were more resistant to multiple carbapenems than other CRE species. Approximately $90 \%$ of CRE isolates in our study were susceptible to tigecycline and colistin, similar to previous studies, ${ }^{1,14}$ but unlike a previous study in Thailand which found only $47 \%$ of CRKP isolates were susceptible to tigecycline. ${ }^{8}$

The main risk factors for CRE acquisition or infection were exposure to healthcare, including admission to the ICU, medical devices, invasive procedures, and antibiotics. ${ }^{15}$ Acquisition of CRKP was associated with poor functional status and ICU stay. ${ }^{16,17}$ Risk factors associated with CRKP infection include recent organ or stem-cell transplantation, receipt of mechanical ventilation, and longer hospital stay. ${ }^{18}$ Although our study found no significant healthcare exposure associated with NSOCE, there is a trend toward a longer ICU stay among NSOCE patients. Several classes of antibiotics have been associated with CRE isolation or infection, including carbapenems, ${ }^{18-20}$ cephalosporins, ${ }^{18}$ fluoroquinolones, ${ }^{16,19}$ and vancomycin. ${ }^{21}$ We found that previous carbapenem exposure was significantly associated with NSOCE patients when compared with NSEE patients. Overall, our hospitalwide carbapenem consumption also increased, with a significant trend over time and with a significant relationship to the incidence of CRE. A meta-analysis found a strong link between antibiotic consumption and antibiotic resistance in the community. The effect of increased antibiotic usage may lead to higher resistance beyond the individual patient level. ${ }^{22}$ Intensive infection control measures, including early identification, rapid patient cohorting, and enhanced contact precautions, were implemented after first detection of a CRE case. Carbapenem restriction has been incorporated into electronic medical prescription at our institution since 2009, which allows empirical carbapenem use for no more than 3 days. Continuation of carbapenems after 3 days without culture-proven ESBL-producing or MDR Gramnegative bacteria must be authorized by infectious diseases or pulmonary physicians. Although our local antibiogram did not show any remarkable decrease in antibiotic susceptibility of Gram-negative bacteria, hospital-wide carbapenem consumption increased notably. One likely explanation is that certain other generic broad-spectrum antibiotics were temporarily unavailable at our institution due to suboptimal quality: cefepime was withdrawn from July to August 2014; cefepime and piperacillin/tazobactam were withdrawn from July to December 2016.

The 30-day all-cause mortality was 14\% higher in the NSOCE group compared to the NSEE group. However, only chronic kidney disease, hematologic malignancies, and SOFA score remained significantly associated with the 30-day all-cause mortality following adjustment in the multivariate model. Although more carbapenems remained available for treatment based on susceptibility testing in the NSEE group, other potential factors, particularly severity of underlying medical conditions, were likely to have a stronger effect on all-cause mortality. ${ }^{23}$ Distinguishing CRE-related mortality from all-cause mortality posed a challenge. Mechanisms of resistance in ertapenem-resistant but imipenem-susceptible $K$. pneumoniae have been demonstrated to be predominantly mediated by non-carbapenemase mechanisms, including defect in porins OmpK35 and OmpK36 expression, ${ }^{11,12} \mathrm{SHV}-$ type ESBLs, and the DHA-1-type AmpC beta-lactamases in comparison to imipenem-non-susceptible $K$. pneumoniae isolates. ${ }^{24} \mathrm{~A}$ study of molecular characterization was done to identify the mechanisms of resistance of NSEE and NSOCE at our institution during 2012-2016. It found that $78 \%$ of the CRE carried carbapenemase genes. The $b l a_{\mathrm{NDM}-1}$ was the most common resistant gene, accounting for almost $70 \%$ of all CRE isolates, followed by the $b l a_{\text {OXA-48 }}$-like group. Only $3 \%$ was $b l a_{\mathrm{IMP}-14}$, and no $b l a_{\mathrm{KPC}}$ was detected. ${ }^{25}$ In another study, only $1.4 \%$ of CRE isolates carried at least one carbapenemase gene, and the most common genes were $b l a_{\mathrm{KPC}-13}$ and $b l a_{\mathrm{IMP}-14 \mathrm{a}}$ in a previous study at another institution in Thailand during 2009-2011. ${ }^{8}$ Although NSEE isolates were phenotypically susceptible to imipenem and meropenem, the underlying 
Table 3 Thirty-day all-cause mortality for adult patients with CRE infections

\begin{tabular}{|c|c|c|c|c|}
\hline Variables & $\begin{array}{l}\text { Unadjusted HR } \\
(95 \% \mathrm{Cl})\end{array}$ & $P$-value & $\begin{array}{l}\text { Adjusted } \mathbf{H R}^{\mathbf{a}} \\
(95 \% \mathrm{Cl})\end{array}$ & $P$-value \\
\hline Non-susceptibility to ertapenem alone & $0.43(0.10-1.90)$ & 0.27 & & \\
\hline Chronic kidney disease & $5.68(1.99-16.25)$ & 0.001 & $4.12(1.02-16.63)$ & 0.046 \\
\hline Solid tumor & $0.31(0.09-1.08)$ & 0.07 & $0.51(0.13-2.05)$ & 0.35 \\
\hline Hematologic malignancies & $6.95(2.6 \mathrm{I}-18.50)$ & $<0.001$ & $6.84(1.86-25.12)$ & 0.004 \\
\hline SOFA score & $\mathrm{I} .28(\mathrm{I} .16-\mathrm{I} .4 \mathrm{I})$ & $<0.001$ & $1.22(I .06-\mid .4 I)$ & 0.007 \\
\hline In ICU at the time of culture & $2.46(0.93-6.47)$ & 0.07 & $1.45(0.43-4.90)$ & 0.55 \\
\hline On mechanical ventilation & $2.57(0.84-7.88)$ & 0.10 & $0.80(0.20-3.21)$ & 0.75 \\
\hline Primary bacteremia & $3.33(1.23-9.03)$ & 0.02 & $1.36(0.39-4.70)$ & 0.63 \\
\hline In vitro active empiric treatment in the first 3 days of infection & $1.97(0.56-6.84)$ & 0.29 & & \\
\hline
\end{tabular}

Notes: ${ }^{a}$ Adjusted for variables with $P<0.10$ in univariate analysis.

Abbreviations: CRE, carbapenem-resistant Enterobacteriaceae; HR, hazard ratio; ICU, intensive care unit; SOFA, Sequential Organ Failure Assessment.

resistance mechanism is important for selecting appropriate antibiotic treatment. ${ }^{26}$ Treatment with carbapenems was not recommended for $b l a_{\mathrm{NDM}-1}$ and $b l a_{\mathrm{OXA}-48}$ producers. ${ }^{26}$ There was $23 \%$ more carbapenem monotherapy in the NSEE group than the NSOCE group, while both groups had similar carbapenemases. This might affect mortality for CRE-infected patients treated with carbapenems in our study. However, our study may have been underpowered due to the small number of subjects enrolled.

This study has several limitations. First, we were unable to complete 30-day follow-up after first CRE detection in 49 patients $(28.1 \%)$, which led to censoring bias and survival bias. We used a Cox model to account for censoring bias. Although we could not eliminate survival bias, the analysis excluding these 49 patients did not significantly change the overall results. Second, as our setting is a tertiary care hospital, it might not be representative of other hospitals with different prevalence of Gram-negative drug resistance. Lastly, comparisons of risk factors and treatment outcome were made as NSEE vs NSOCE. Further comparisons of CRE vs non-CRE patients may illustrate more distinctions of different patterns of antibiotic resistance.

\section{Conclusion}

The incidence of CRE has risen significantly over a 5-year period at our institution. The important risk factor for non-susceptibility to other carbapenems compared to nonsusceptibility to ertapenem alone was previous carbapenem use. Our hospital-wide carbapenem use has significantly increased over time, and is associated with the increasing incidence of CRE. Emphasis on antimicrobial stewardship could be an effective strategy to slow down the rising CRE trends.

\section{Acknowledgments}

We thank the infection control team for monitoring infections and collecting data. This work received no specific funding and was performed at the Faculty of Medicine Ramathibodi Hospital, Mahidol University. Partial results of this study were presented as a poster presentation at IDWeek 2018, October 3-7, 2018 (San Francisco, CA, USA).

\section{Disclosure}

The authors report no conflicts of interest in this work.

\section{References}

1. Guh AY, Bulens SN, Mu Y, et al. Epidemiology of carbapenemresistant Enterobacteriaceae in 7 US communities, 2012-2013. JAMA. 2015;314(14):1479-1487.

2. Falagas ME, Tansarli GS, Karageorgopoulos DE, Vardakas KZ. Deaths attributable to carbapenem-resistant Enterobacteriaceae infections. Emerg Infect Dis. 2014;20(7):1170-1175.

3. Fraenkel-Wandel Y, Raveh-Brawer D, Wiener-Well Y, Yinnon AM, Assous MV. Mortality due to blaKPC Klebsiella pneumoniae bacteraemia. J Antimicrob Chemother. 2016;71(4):1083-1087.

4. Gutiérrez-Gutiérrez B, Salamanca E, de Cueto M, et al; REIPI/ESGBIS/INCREMENT Investigators. Effect of appropriate combination therapy on mortality of patients with bloodstream infections due to carbapenemase-producing Enterobacteriaceae (increment): a retrospective cohort study. Lancet Infect Dis. 2017;17(7):726-734.

5. Marimuthu K, Venkatachalam I, Khong WX, et al; CarbapenemaseProducing Enterobacteriaceae in Singapore (CaPES) Study Group Clinical and molecular epidemiology of carbapenem-resistant Enterobacteriaceae among adult inpatients in Singapore. Clin Infect Dis. 2017;64(Suppl 2):S68-S75.

6. Zhang Y, Wang Q, Yin Y, et al. Epidemiology of carbapenem-resistant Enterobacteriaceae infections: report from the China CRE network. Antimicrob Agents Chemother. 2018;62(2):e01882-e01917.

7. Tzouvelekis LS, Markogiannakis A, Psichogiou M, Tassios PT, Daikos GL. Carbapenemases in Klebsiella pneumoniae and other Enterobacteriaceae: an evolving crisis of global dimensions. Clin Microbiol Rev. 2012;25(4):682-707.

8. Netikul T, Kiratisin P. Genetic characterization of carbapenem-resistant Enterobacteriaceae and the spread of carbapenem-resistant Klebsiella pneumonia ST340 at a university hospital in Thailand. PLoS One. 2015;10(9):e0139116. 
9. Rimrang B, Chanawong A, Lulitanond A, et al. Emergence of NDM-1and IMP-14a-producing Enterobacteriaceae in Thailand. J Antimicrob Chemother. 2012;67(11):2626-2630.

10. Meletis G. Carbapenem resistance: overview of the problem and future perspectives. Ther Adv Infect Dis. 2016;3(1):15-21.

11. Jacoby GA, Mills DM, Chow N. Role of beta-lactamases and porins in resistance to ertapenem and other beta-lactams in Klebsiella pneumoniae. Antimicrob Agents Chemother. 2004;48(8):3203-3206.

12. Chung HS, Yong D, Lee M. Mechanisms of ertapenem resistance in Enterobacteriaceae isolates in a tertiary university hospital. J Investig Med. 2016;64(5):1042-1049.

13. Clinical and Laboratory Standards Institute. Performance standards for antimicrobial susceptibility testing; twenty-third informational supplement. M100-S123. Wayne, PA: CLSI; 2013.

14. Han JH, Goldstein EJ, Wise J, Bilker WB, Tolomeo P, Lautenbach E. Epidemiology of carbapenem-resistant Klebsiella pneumoniae in a network of long-term acute care hospitals. Clin Infect Dis. 2017;64(7):839-844.

15. Gupta N, Limbago BM, Patel JB, Kallen AJ. Carbapenem-resistant Enterobacteriaceae: epidemiology and prevention. Clin Infect Dis. 2011;53(1):60-67.

16. Schwaber MJ, Klarfeld-Lidji S, Navon-Venezia S, Schwartz D, Leavitt A, CarmeliY. Predictors of carbapenem-resistant Klebsiella pneumoniae acquisition among hospitalized adults and effect of acquisition on mortality. Antimicrob Agents Chemother. 2008;52(3):1028-1033.

17. Ripabelli G, Tamburro M, Guerrizio G, et al. Tracking multidrugresistant Klebsiella pneumoniae from an Italian hospital: molecular epidemiology and surveillance by PFGE, RAPD and PCR-based resistance genes prevalence. Curr Microbiol. 2018;75(8):977-987.

18. Patel G, Huprikar S, Factor SH, Jenkins SG, Calfee DP. Outcomes of carbapenem-resistant Klebsiella pneumoniae infection and the impact of antimicrobial and adjunctive therapies. Infect Control Hosp Epidemiol. 2008;29(12):1099-1106.
19. Hussein K, Sprecher H, Mashiach T, Oren I, Kassis I, Finkelstein R. Carbapenem resistance among Klebsiella pneumoniae isolates: risk factors, molecular characteristics, and susceptibility patterns. Infect Control Hosp Epidemiol. 2009;30(7):666-671.

20. Jeon MH, Choi SH, Kwak YG, et al. Risk factors for the acquisition of carbapenem-resistant Escherichia coli among hospitalized patients. Diagn Microbiol Infect Dis. 2008;62(4):402-406.

21. Wiener-Well Y, Rudensky B, Yinnon AM, et al. Carriage rate of carbapenem-resistant Klebsiella pneumoniae in hospitalised patients during a national outbreak. J Hosp Infect. 2010;74(4):344-349.

22. Bell BG, Schellevis F, Stobberingh E, Goossens H, Pringle M. A systematic review and meta-analysis of the effects of antibiotic consumption on antibiotic resistance. BMC Infect Dis. 2014;14(1):13.

23. Viale P, Giannella M, Lewis R, Trecarichi EM, Petrosillo N, Tumbarello M. Predictors of mortality in multidrug-resistant Klebsiella pneumoniae bloodstream infections. Expert Rev Anti Infect Ther. 2013;11(10): 1053-1063.

24. Wu JJ, Wang LR, Liu YF, Chen HM, Yan JJ. Prevalence and characteristics of ertapenem-resistant Klebsiella pneumoniae isolates in a Taiwanese university hospital. Microb Drug Resist. 2011;17(2): 259-266.

25. Laolerd W, Akeda Y, Preeyanon L, Ratthawongjirakul P, Santanirand P. Carbapenemase-producing carbapenem-resistant Enterobacteriaceae from Bangkok, Thailand, and their detection by the Carba NP and modified carbapenem inactivation method tests. Microb Drug Resist. 2018;24(7):1006-1011.

26. Hawkey PM, Warren RE, Livermore DM, et al. Treatment of infections caused by multidrug-resistant Gram-negative bacteria: report of the British Society for Antimicrobial Chemotherapy/Healthcare infection Society/British Infection Association joint Working Party. J Antimicrob Chemother. 2018;73(Suppl 3):iii2-iii78.
Infection and Drug Resistance

\section{Publish your work in this journal}

Infection and Drug Resistance is an international, peer-reviewed openaccess journal that focuses on the optimal treatment of infection (bacterial, fungal and viral) and the development and institution of preventive strategies to minimize the development and spread of resistance. The journal is specifically concerned with the epidemiology of antibiotic
Dovepress

resistance and the mechanisms of resistance development and diffusion in both hospitals and the community. The manuscript management system is completely online and includes a very quick and fair peerreview system, which is all easy to use. Visit http://www.dovepress.com/ testimonials.php to read real quotes from published authors. 